Pacific Journal of Mathematics

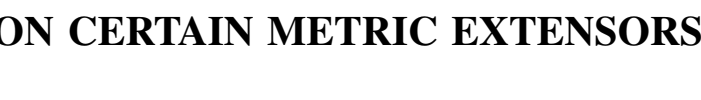




\title{
ON CERTAIN METRIC EXTENSORS
}

\author{
Homer V. Craig and B. B. Townsend
}

1. Introduction. A straight line-segment (the basis of the fundamental entity of classical vector analysis) is perhaps the simplest nontrivial example of a continuous flat space. Furthermore, it seems that, from an elementary point of view, a higher space should be regarded as completely flat only if it is composed of these elementary flat spaces (strokes) in such a way that the vector $\Delta \vec{\rho}$ joining any two points of the higher space lies entirely in that space. A criterion of similar spirit is the requirement that no such $\Delta \vec{\rho}$ have a component normal to the space. Since we prefer to regard a plane patch with a concavity in the boundary curve as being "completely flat", we adopt (for heuristic purposes) the second test. Either view leads quite naturally to the investigation of certain immersed spaces $R_{N}$ from the standpoint of the various derivatives of the radius vector $\vec{\rho}$ since these local quantities determine $\Delta \vec{\rho}$. With regard to the space $R_{N}$, we assume that it is a Riemannian space defined vectorially by means of the equation

$$
\vec{\rho}=\vec{\rho}\left(x^{1}, x^{2}, \cdots, x^{N}\right),
$$

with $\vec{\rho}$ the radius-vector from an origin in an enveloping classical vector-space to a generic point of $R_{N}$. The function $\vec{\rho}(x)$ is assumed to be such that $\Delta \vec{\rho}$ can be determined by a Taylor's expansion. Obviously, the term completely flat as used here is applicable to straight lines and planes but not to certain intrinsically flat spaces (i.e., flat in the sense that the Riemann-Christoffel tensor vanishes), such as curved lines and cylinders. The basic datum here is the Euclidean vector-space with it directed strokes which can be used to test lines and other immersed spaces for complete flatness.

Since $\Delta \vec{\rho}$ is determined by the various derivatives of $\vec{\rho}$ at the point of issue of $\Delta \vec{\rho}$, it is obvious that complete flatness or deviation therefrom will show up in the derivatives of $\vec{\rho}$. These vector derivatives may have components normal to $R_{N}$ and hence, in this case, do not belong to $R_{N}$ in a strong sense. However, by forming certain dot products, they can be converted to scalar functions which may more appropriately by assigned to the immersed space. Among these 
products are extensors which contain such important quantities as the metric tensor and the Christoffel symbols and therefore seem worthy of study. Accordingly, we have taken as the primary purpose of this paper the investigation of the geometrical, algebraic, and differential properties of certain extensors which are immediately derivable from the derivatives of $\vec{\rho}$.

In developing these extensors, we shall not restrict our attention entirely to curves and a single parameter, but instead consider two-dimensional spreads involving two parameters. Kawaguchi has already introduced a highly satisfactory theory of extensors based on several paranieters [8]. However, to secure more unity in the present paper, we shall deviate from Kawaguchi's notation and designate partial derivatives of certain base letters by means of "matrix primes". A description of this notation and a brief account of the theory of matrix extensors sufficient for applications in this paper will be presented in the next two sections.

2. Notation. The symbolism to be employed in the present paper is essentially that used in preceding works on the subject (see [9] and [1-6]), except that Greek indices will be used both to denote integers, 0 to $M$, as before, and to denote matrices. To illustrate, if the coordinate variables $x^{a}$ are made functions of a single parameter $t$, the usual case, then as before $x^{(\alpha) a}$ and $x^{a(\alpha)}$ will be used interchangeably to denote $d^{\alpha} x^{a} / d t^{a}$, while $X_{\alpha a}^{\rho r}$ and $X_{\rho r}^{\alpha a}$ will represent $\partial x^{(\rho) r} / \partial x^{(\alpha) a}$ and $\partial x^{(\alpha) a} / \partial x^{(\rho) r}$, respectively--indices $a, b, c, \cdots$ at the first of the alphabet indicate coordinate system $x$, while $r, s, t, \ldots$ are to be correlated to system $\bar{x}$. However, if the $x^{a}$ are functions of two parameters $u$ and $v$, and $\alpha$ represents the matrix $(2,3)$, for example, then $x^{(\alpha) a}$ and $x^{a(\alpha)}$ will each stand for $\partial^{5} x^{a} / \partial^{2} u \partial^{3} v$. Here the first element 2 in the matrix prime $(2,3)$ indicates the order of differentiation with respect to the first parameter, etc. Similarly, if $\alpha$ and $\rho$ have the matrix values $\left(a_{1}, a_{2}\right)$ and $\left(\rho_{1}, \rho_{2}\right)$, respectively, then $X_{(\rho) r}^{(a) a}$ is to be interpreted as the partial derivative of $x^{(\alpha) a}$ with respect to $x^{(\rho) r}$ as before, except that $x^{(a) a}$ now represents the result of differentiating $x^{a}$ partially $\alpha_{1}$ times with respect to the first parameter $u$ and $\alpha_{2}$ times with respect to $v$, while $x^{(\rho) r}$ is to be interpreted similarly. Evidently,

$$
x^{(\alpha) a(\beta)}=x^{(\alpha+\beta) a},
$$

with the symbol + indicating matrix addition. Summations, on repeated lower case Greek indices, unless the contrary is indicated, will be from zero to $M$ in the case of one parameter, and over the set of matrices $\left(a_{1}, a_{2}\right)$ with $a_{1}$ ranging from 0 to $M_{1}$ and $\alpha_{2}$ from 0 to $M_{2}$. In addition, "matrix binomial coefficients" $\left(\begin{array}{l}a \\ \beta\end{array}\right), a=\left(\alpha_{1}, a_{2}\right), \beta=\left(\beta_{1}, \beta_{2}\right)$, will be used to denote the product of the 
binomial coefficients obtained from columns in the symbol $\left(\begin{array}{l}\alpha \\ \beta\end{array}\right)$, i. e., by definition,

$$
\left(\begin{array}{l}
\alpha \\
\beta
\end{array}\right)=\left(\begin{array}{l}
\alpha_{1} \\
\beta_{1}
\end{array}\right)\left(\begin{array}{l}
a_{2} \\
\beta_{2}
\end{array}\right)
$$

3. Matrix extensors. The foundation for the theory of matrix extensors is of course the transformation equation which relates the components in one system $(x)$ to those in another system $(\bar{x})$. This equation, which is exemplified by the relationship

$$
T_{\beta b}^{\alpha a}=T_{\sigma s}^{\rho r} X_{\rho r}^{\alpha a} X_{\beta b}^{\sigma s}
$$

is formally equivalent to that of tensor and extensor analysis. The essential points of difference are that the Greek indices now represent matrices, and the coordinate variables have been made functions of two or more parameters ${ }^{1}$.

To obtain a simple example of a matrix extensor, let the coordinate variables $x^{a}$ be made functions of the paraneters $u$ and $v$, and let $f$ be a function of the $x^{(a) a}$, with $a$ running through the set of matrices $\left(c_{v_{1}}, v_{v_{2}}\right)$ for which $a_{1}$ and $c_{2}$ have ranges 0 to $M_{1}$ and 0 to $M_{2}$, respectively. If we denote by $\bar{f}$ the function of the $\bar{x}$ 's obtained by replacing each of the variables $x^{(\alpha) a}$ in $f$ by its value in terms of the barred coordinates as determined by the coordinate transformations

$$
x^{a}=x^{a}(\bar{x}), x^{r}=x^{r}(x),
$$

and take $\bar{f}$ as the $\bar{x}$ mate of $f$, then we have an instance of an absolute invariant, and may write $f=\bar{f}$. Differentiating the left member directly with respect to $x^{(\boldsymbol{a}) a}$ and the right member through the $x^{(\rho) r}$ as intermediate variables, we obtain

$$
f_{; \alpha a}=f_{; \rho r} X_{\alpha a}^{\rho r}
$$

Hence, as in the one-parameter case, $f_{; \alpha a}$ is an extensor.

A successive application of the matrix differentiators $\left(M_{1}, 0\right)$ and $\left(0, M_{2}\right)$ to a product of two functions $F$ and $G$ with application of the Leibnitz rule of differentiation gives

$$
\begin{aligned}
& {\left[(F G)^{\left(M_{1}, 0\right)}\right]^{\left(0, M_{2}\right)}=\left[\sum_{\alpha_{1}=0}^{M_{1}}\left(\begin{array}{c}
M_{1} \\
a_{1}
\end{array}\right) F^{\left(\alpha_{1}, 0\right)} G^{\left(M_{1}-a_{1}, 0\right)}\right]\left(0, M_{2}\right)} \\
& =\sum_{\alpha_{2}=0}^{M_{2}} \sum_{\alpha_{1}=0}^{M_{1}}\left(\begin{array}{c}
M_{1} \\
\alpha_{1}
\end{array}\right)\left(\begin{array}{c}
M_{2} \\
a_{2}
\end{array}\right)\left(F^{\left(\alpha_{1}, 0\right)}\right)^{\left(0, \alpha_{2}\right)}\left(G^{\left(M_{1}-a_{1}, 0\right)}\right)^{\left(0, M_{2}-\alpha_{2}\right)} \text {, }
\end{aligned}
$$

${ }^{1}$ An incomplete study of matrix extensors by H. V. Craig and a subsequent check by J. C. Evans has shown that most of the properties of the one-parameter extensors carry over without formal change to the multiple-parameter case. 
or

$$
(F G)^{(M)}=\sum_{a=0}^{M}\left(\begin{array}{c}
M \\
\alpha
\end{array}\right) F^{(a)} G^{(M-\alpha)},
$$

and the form of the Leibnitz rule for the differentiation of a product is the same as in the one-parameter case.

Similarly, the commutation-reduction formula,

$$
F_{j a a}^{(M)}=\left(\begin{array}{c}
M \\
\boldsymbol{a}
\end{array}\right) F_{\boldsymbol{a}}^{\cdot(M-A)}
$$$$
A=a=\left(a_{1}, a_{2}\right)
$$

may be established by following the procedure used in the one-parameter case. See $[1$, p. 457 and 3, p. 215$]$. Consequently, we have

$$
X_{\rho r}^{\alpha a}=\left(\begin{array}{l}
A \\
P
\end{array}\right) X_{r}^{a(A-P)}, \quad A=c_{v}=\left(c_{1}, a_{2}\right), P=\rho=\left(\rho_{1}, \rho_{2}\right) .
$$

It now becomes immediately obvious that if $V^{a}$ is a contravariant tensor then $V^{a(\alpha)}$ is a matrix extensor, for

$$
V^{a(\alpha)}=\left(V^{r} X_{r}^{a}\right)^{(\alpha)}=\sum_{\rho=0}^{a}\left(\begin{array}{l}
a \\
\rho
\end{array}\right) V^{r(\rho)} X_{r}^{a(\alpha-\rho)}=V^{r(\rho)} X_{\rho r}^{\alpha a} .
$$

4. The fundamental metric extensors. Given the Riemannian space $R_{N}$ introduced in the first section, there are at least three methods for generating extensors having the property that they contain among their components the fundamental metric tensor and the associated two-index components of connection,

$$
[a, c]\left(=[a b, c] x^{b^{\prime}}\right) \text { and }\left\{\begin{array}{l}
a \\
c
\end{array}\right\}\left(=\left\{\begin{array}{c}
a \\
b c
\end{array}\right\} x^{b^{\prime}}\right)
$$

quantities which are derivable from the radius vector $\vec{\rho}$. Because of the obvious and well-known metrical properties of the $g^{\prime} s$, and the fact that the Christoffel symbols are involved in the equations for geodesics and the equipollent displacement of vectors, matters baving direct metrical implications, it seems appropriate to refer to these extensors collectively as metric extensors. The components of two of these extensors are of the nature of higher order components of connection; and we shall distinguish between them, for reasons which will be presented later, by referring to them as the direct and alternating components of connection.

The direct connection extensor $g_{\alpha a}^{b}$. As a preliminary to the development of the direct extensor $g_{a a}^{b}$, let us note that the part of $\Delta \vec{\rho}$ which belongs to the com- 
pletely flat tangent space $T_{N}$ is given by the invariant expression

$$
\Delta \vec{\rho} \cdot \vec{\rho}^{b} \vec{\rho}_{b} \quad\left(\vec{\rho}_{b}=\frac{\partial \vec{\rho}}{\partial x^{b}}, \vec{\rho}^{b}=g^{a b} \vec{\rho}_{a}\right),
$$

and that $\rfloor \vec{\rho}$ may be written in the form

$$
\Delta \vec{\rho}=\Delta \vec{\rho} \cdot \vec{\rho}^{b} \vec{\rho}_{b}+\vec{\nu}
$$

in which $\vec{v}$ is normal to $T_{N}$ in the sense that

$$
\vec{\nu} \cdot \vec{\rho}^{c}=\vec{\nu} \cdot \vec{\rho}_{c}=0
$$

To establish this last relaticnship, it suffices to multiply the preceding equation by $\vec{\rho}^{c}$, and note that $\vec{\rho}^{c} \cdot \vec{\rho}_{b}=\delta_{b}^{c}$. See [3, p. 202-203]. In order for our space to be completely flat in the sense of the foregoing definition, it is necessary and sufficient that $\vec{\nu}=0$ for all $\vec{\Delta} \vec{\rho}$ 's, which means of course that the quantities $\Delta \vec{\rho} \cdot \vec{\rho}^{b}$ constitute a complete set of components for $\Delta \vec{\rho}$. Expanding $\Delta \vec{\rho}$ in a power series, and noting that

$$
\vec{\rho}^{(M+1)}=\vec{\rho}_{; \alpha a}^{(M)} x^{(\alpha+1) a}, \quad\left(\vec{\rho}_{; \alpha a}^{(M)} \equiv \frac{\partial \vec{\rho}^{(M)}}{\partial x^{(\alpha) a}}\right)
$$

we see that, whether $R_{N}$ is flat or not,

$$
\Delta \vec{\rho} \cdot \vec{\rho}^{b}=\sum_{M=0}^{\infty} \vec{\rho}_{; \alpha a}^{(M)} \cdot \vec{\rho}^{b} x^{\cdot(\alpha) a} \frac{t^{M+1}}{(M+1) !}
$$

Hence if we adopt:

ĐE FINITION 4.1. $g_{a a}^{b}=\vec{\rho}_{; \alpha a}^{(M)} \cdot \vec{\rho}^{b}$, then we may assert:

THEOREM 4.1. If $P$ and $Q$ are any two points of $R_{N}, \Delta \vec{\rho}$ their joining vector, and $C$ is any analytic parameterized arc joining $P$ and $Q$, then

$$
\Delta \vec{\rho} \cdot \vec{\rho}^{b}=\sum_{M=0}^{\infty} g_{a a}^{b} x^{\cdot(a) a} \frac{t^{M+1}}{(M+1) !}
$$

That is, the quantities $g_{\alpha a}^{b}$, which obviously are extensors have the property that they determine (through contraction with the extensor $x^{\prime(a) a}$ ) the coefficients in the fundamental power series (4.2). Thus they obviously play a basic 
role in the determination by means of curves in the imbedded space $R_{N}$ (intrinsic determination) of the part of $\lambda \vec{\rho}$ that belongs to $R_{N}$ in the sense of belonging to the tangent space $T_{N}$. As noted previously (see [3, pp.279-281]), the $g_{a a}^{b}$ contain the Kronecker delta $\delta_{a}^{b}$ and the two-index Christoffel symbols $\left\{\begin{array}{l}b \\ a\end{array}\right\}$. Furthermore, the result of contracting $g_{a a}^{b}$ with $V^{a(a)}$ for $M^{\prime}=1$ is $l V^{b}$ - the intrinsic derivative of $V^{b}$. In general, if $\vec{\sigma}$ denotes $V^{a} \vec{\rho}_{a}$, the vector with scalar components $l^{\prime a}$, then our contraction $V^{a(a)} g_{a a}^{b}$ equals $\vec{\sigma}^{(M)} \cdot \vec{\rho}^{b}$, which we recognize as the scalar components of the part of $\vec{\sigma}^{(M)}$ which belongs to $T_{N}$. Thus we see again that the extensor $g_{a a}^{b}$ is involved in the intrinsic geometry of $R_{N}$, and that, since, for $M=1, I V^{b}=\vec{\sigma}^{(M)} \cdot \vec{\rho}^{b}$, there is justification in regarding $V^{a(a)} g_{a a}^{b}$ as a kind of higher order intrinsic derivative. Since derivatives of $\vec{\sigma}$ may not belong to $T_{N}$, it is perhaps more appropriate to think of higher order derivatives $\vec{\sigma}^{(M)}$ as being computed ( see [7, p. 573]) directly from the values of $\vec{\sigma}$ at a set of points converging to the given point instead of by a process of successive differentiation.

The alternating connection extensor $L_{a a}^{b}$. From the viewpoint of the enveloping space, intrinsic differentiation in the usual sense is a process which applied to the scalar components of a vector $\vec{\sigma}$ (directed stroke) in $T_{N}$ yields the scalar components of the part of $\vec{\sigma}^{\prime}$ which belongs to $T_{N}$. The strict iteration of this process consists in computing the components of the part of the derived vector which belongs to $T_{N}$ after each differentiation. Thus the steps in the iterated process are as follows: given $V^{a}$ construct $\vec{\sigma}$,

$$
\vec{\sigma}=V^{a} \vec{\rho}_{a}
$$

differentiate $\vec{\sigma}$, and compute $\vec{\sigma}^{\prime} \cdot \vec{\rho}^{b}$ (the components of the part of $\vec{\sigma}^{\prime}$ that belongs to the space $T_{N}$ ). The result is the intrinsic derivative, $I V^{b}$. The strict iteration of this process consists in replacing the original $V^{b}$ with $l V^{b}$ and proceeding as before. The extensor $L_{a a}^{b}$ (together with its mate $L_{b}^{a a}$ ), which plays the role of $g_{a a}^{b}$ in this iterated process, is derivable from the extensor $g_{a a}^{b}, M=1$, by a process called extensive differentiation. See [4, p.24-29]. This process involves the nontensor component of $g_{\alpha a}^{b}(a=0)$ and generates the new quantities by repeated application to the tensor components $(a=1), \delta_{a}^{b}$. These extensors have been called the extended components of connection.

The extensors $g_{a a}^{b}$ and $L_{a a}^{b}$ coincide in the ranks $c=M, a=M-1$; otherwise, for $M>1$ they are distinct. The primary difference between their associated derivatives $g_{a a}^{b} V^{a(a)}$ and $L_{x a}^{b} V^{a(x)}$ is that the former may be obtained by first computing the derivative $\left(V^{a} \vec{\rho}_{a}\right)^{(M)}$ directly and then selecting the components of the part that belongs to $T_{N}$, while the latter involves an alternation 
of the process of "priming", and "dotting" into the base vectors $\vec{\rho}^{b}$. It is for this reason that we have adopted the terms direct and alternating to distinguish the extensors of this section.

Since

$$
\vec{\rho}_{; \propto a}^{(M)}=\left(\begin{array}{c}
M \\
A
\end{array}\right) \vec{\rho}_{a}^{\cdot(M-A)},
$$

and is thus the simplest extensor obtainable by priming the base vectors $\vec{\rho}_{a}$, while $\vec{\rho}^{a(\alpha)}$ is the simplest extensor obtainable by priming $\vec{\rho}^{a}$, it seenis that the quantities $g_{b}^{\alpha a}$ defined by $g_{b}^{\alpha a}=\vec{\rho}^{a(a)} \cdot \vec{\rho}_{b}$ are in a sense the mate quantities of $g_{x a}^{b}$ obtainable by interchanging the role of $\vec{\rho}^{a}$ and $\vec{\rho}_{a}$. This immediately raises the question of the construction of suitable extensors $g_{a a \beta b}, g^{a a, \beta b}$ for raising and lowering doublet indices: a problem which introduces the third set of metric extensors and to which we shall turn presently.

In passing, we note that both $g_{b}^{\alpha a}$ and $g_{\alpha a}^{b}$ are included in the extensor $\vec{\rho}^{a(a)}$. $\vec{\rho}_{; \beta b}^{(. h)}$ denoted by $g_{\beta b}^{a a}$. Other quantities which should be grouped with these from the structural viewpoint are the extensors $g^{\alpha a \beta b}$ and $g_{\alpha a \beta b}$ which are defined as follows:

DEFINITION 4.2. $g^{\alpha a \beta b}=\vec{\rho}^{a(a)} \cdot \vec{\rho}^{b(\beta)}, g_{a a \beta b}=\vec{\rho}_{; \alpha a}^{(M)} \cdot \vec{\rho}_{; \beta b}^{(M)}$.

These last two extensors contain the metric tensors $g_{a b}$ and $g^{a b}$ and the twoindex Christoffel symbols $[a b, c] x^{c^{\prime}}$.

The interchange extensors. To complete the introduction of the metric extensors, we return to the problem of the construction of the extensors to be used in raising and lowering doublet indices (i.e., in interchanging contravariance and covariance). We shall see presently that a satisfactory formulation is given by:

1) E F INITION 4.3. $g_{\alpha a \beta b}=\left(\begin{array}{c}M \\ A, B\end{array}\right) g_{a b} \ddot{a(M-A-B)}, g^{\alpha a \beta b}=\left[\begin{array}{c}A B \\ M\end{array}\right] g^{a b(A+B-M)}$, $A=\alpha, B=\beta$.

The symbols $(\underset{A, B}{M}),\left[\begin{array}{c}A B \\ M\end{array}\right]$ are given in $[5, \mathrm{p} .334$; see also p. $335-336$, Theorems ( 2.1$),(2.3)$.

The prime requirement of an interchange extensor is that it have a kind of group property so that the successive raising and lowering (or vice versa) of a given index leaves the extensor unaltered. This characteristic for the quantities just defined is ensured by: 
THEOREM 4.2. $g_{\alpha a \beta b} 9^{a a \gamma c}=\delta_{\beta}^{\gamma} \delta_{b}^{c}$.

Proof. Expanding

$$
Q \equiv\left(\begin{array}{l}
\Gamma \\
B
\end{array}\right)\left(g_{a b} g^{a c}\right)^{(\Gamma-B)},
$$

which is obviously equal to the right member in the relationship to be established, we get

$$
Q=\left(\begin{array}{c}
\Gamma \\
B
\end{array}\right) \sum_{a=0}^{\Gamma-B}\left(\begin{array}{c}
\Gamma-B \\
\alpha
\end{array}\right) g_{a b}^{(\alpha)} g^{a c(\Gamma-B-\alpha)} .
$$

If we now replace $c_{0}$ according to the relationship $a_{c}=M !-\bar{c}_{0}-B$, subsequently drop the bar, and then make use of the formula

$$
\left(\begin{array}{c}
\Gamma \\
B
\end{array}\right)\left(\begin{array}{c}
\Gamma-B \\
M-A-B
\end{array}\right)=\left[\begin{array}{c}
A, \Gamma \\
M
\end{array}\right]\left(\begin{array}{c}
M \\
A B
\end{array}\right),
$$

there results the $\operatorname{sum} \sum_{9_{\alpha a \beta b}} g^{\alpha a \gamma c}$, with summation on $C$. from $M-y$ to $M-3$. This range, however, nay be changed to the regular range $0, M$, since

$$
\left(\begin{array}{c}
M \\
\alpha, \beta
\end{array}\right)=0 \text { for } c_{0}>M-\beta,
$$

while

$$
\left[\begin{array}{c}
a, \gamma \\
M
\end{array}\right]=0 \text { when } a<\|-\gamma \text {. }
$$

REMARK. The quantities $g$ of Definition 4.3 are obviously symmetric in the doublet indices; hence their order in Theorem 4.2 is not essential. Furthermore, the excontravariant $g$ 's given in Definition 4.3 are the only symmetric quantities that will satisfy this theorem if the choice of $g_{\alpha a \beta b}$ has been fixed as in this definition. To check this point, we may assume a separate set $G$ sup $\beta b \delta d$ and multiply $G$ into the equation presented in Theorem 4.2. In the following section we shall see that the script $g$ 's have ideal properties relative to the other metric extensors. They obviously contain the metric tensors $g^{a b}$ and $g_{a b}$ among their components.

5. Interchanges. We shall refer to any two extensors $T_{\ldots} \ldots, T_{\ldots} \ldots$ which are related in accordance with the equivalent equalities

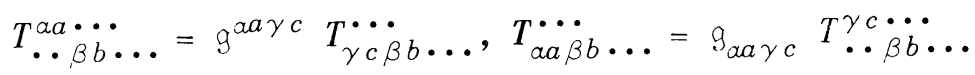

as interchanges of each other. Ne shall now show that certain pairs of quantities which are otherwise natural mates are pairs of interchanges. We may if we like 
regard the members of each pair as constituting different descriptions of the same extensor.

THEOREM 5.1. If

$$
A^{a a}=A^{a(\alpha)} \text { and } A_{\alpha a}=\left(\begin{array}{c}
M \\
A
\end{array}\right) A_{a}^{\cdot(M-A)},
$$

then $A^{\alpha a}$ and $A_{\alpha a}$ are interchanges.

Proof. For arbitrary quantities $L^{b}$,

$$
\begin{gathered}
\sum_{a, \beta}\left(\begin{array}{c}
M \\
a, \beta
\end{array}\right)\left(g_{a b}\right)^{(M-\alpha-\beta)} A^{a(\alpha)} B^{b(\beta)}=\left(g_{a b} A^{a} B^{b}\right)^{(M)} \\
=\sum\left(\begin{array}{c}
M \\
\beta
\end{array}\right) A_{b}^{(M-\beta)} B^{b(\beta)}=A_{\beta b} B^{b(\beta)} .
\end{gathered}
$$

REMARK. The first equality of the foregoing chain tells us the meaning of the invariant $g_{\alpha a \beta b} A^{a(\alpha)} B^{b(\beta)}$; this quantity is the $M /$ th derivative of the dot product of $A^{a}$ and $B^{b}$.

REMARK. The proof of this theorem is valid in case $A^{a}$ represents a set of directed strokes instead of a set of scalars. Hence, $\vec{\rho}^{a(\alpha)}$ and $\vec{\rho}_{; \alpha a}^{(M)}$ are interchanges, since

$$
\vec{\rho}_{; \alpha a}^{(M)}=\left(\begin{array}{c}
M \\
A
\end{array}\right) \vec{\rho}_{a}^{\cdot(M-A)}, A=u .
$$

THEOREM 5.2. The extensors $g_{b}^{a a}$ and $g_{\alpha a}^{b}$ are interchanges, as are the quantities $g_{a a \beta b}, g^{\alpha a \beta b}$ of Definition 4.2 .

Proof. We have

$$
g_{\alpha a}^{b}=\vec{\rho}^{b} \cdot \vec{\rho}_{; \alpha a}^{(M)}=g^{b d} \vec{\rho}_{d} \cdot \vec{\rho}^{c(\gamma)} g_{\gamma c \alpha a}=g^{b d} g_{\gamma c \alpha a} g_{d}^{\gamma c} \cdot
$$

The proof of the second part is similar to the foregoing.

REMARK. The extensors $g_{a a \beta b}, g^{a a \beta b}$ themselves are obviously interchanges because of the Kronecker delta relationship expressed by Theorem 4.2.

THEOREM 5.3. The two alternating components of connection $L_{a a}^{b}$ and $L_{b}^{a a}$ constitute a pair of interchanges.

Proof. From the relationship 


$$
g^{\alpha a \beta b}=\sum_{\delta=0}^{a+\beta-M}\left\{\begin{array}{l}
\alpha, \beta \\
M, \delta
\end{array}\right\} g^{f h} L_{f}^{\delta b} L_{h}^{\alpha+\beta-M-\delta \cdot a},
$$

which is a corollary of the independent proposition '7heoren: 6.3 , we derive at once by "wrultiplying" by $g_{d c} L_{a c}^{c}$ and extending the range of $\delta$ to 0 , $M$, the equality

$$
g_{d c} g^{x a \beta b} L_{a a}^{c}=\sum_{a, \delta}\left\{\begin{array}{c}
\alpha, \beta \\
i, \delta
\end{array}\right\} g^{f h} g_{d c} L_{\alpha a}^{c} L_{\dot{h}}^{a+\beta-M-\delta \cdot \alpha} L_{f}^{\delta b} .
$$

Now

$$
\{\underset{M, \delta}{a, \beta}\}=(\underset{M+\Delta-\beta}{a}) \frac{B !(M+\Delta-B) !}{(M ! \Delta !)}, \quad B=\beta, \Delta=\delta,
$$

and since the coefficient \{\} vanishes when $a<M+\delta-\beta$, the part of the right member of our equality which involves 0 . may be written in the form

$$
\left(R L_{h}^{a-(M+\Delta-\beta) \cdot \alpha} L_{\alpha a}^{c}\right) \frac{B !(A i+\Delta-B) !}{(1 ! ! \Delta !)}
$$

Here, as before, the capitals $B$ and $\Delta$ have been introduced for $\beta$ and $\delta$ to emphasize that these letters are not to be summed; and $R$ stands for the symbolism

$$
\sum_{\alpha=M+\Delta-\beta}^{M}(\underset{M+\Delta-B}{a})
$$

This abridgement is possible because the pattern of letters in the binomial coefficient and at the base of the summation sign can be generated from the Greek superscript $u_{-}-(M+\Delta-B)$. See $[6, \mathrm{p} .234]$. We next note that according to Theorem 7.1 the contracted product of the $L$ 's vanishes excepting when $\delta=\beta$, in which case the contraction collapses to $\delta_{h}^{a} \delta_{a}^{c}$ or $\delta_{h}^{c}$, and the factorial multiplier reduces to unity. Thus the right member of $(\overline{5.2})$ is equal to $L_{d}^{\beta b}$, and the proof is complete.

REMARK. The proof of this theorem points to the desirability for a study of the contractions of our metric extensors-a matter which we shall consider presently.

6. The derivatives of equipollent tensors. An immediately obvious but nevertheless useful minor proposition of tensor analysis asserts that if 


$$
I T^{a}=0
$$

then

$$
T^{a^{\prime}}=T^{b}\left(-L_{b}^{a}\right), L_{b}^{a}=\left\{\begin{array}{c}
a \\
b c
\end{array}\right\} x^{\prime c} .
$$

Furthermore, from the structure of the intrinsic derivative of a higher order tensor, it is readily apparent that all derivatives of any equipollent tensor $(I T=0)$ are expressible as linear forms in the components of $T$ with coefficients that are built up of derivatives and products of $L$ 's. The problem then arises of deternining the composition of these coefficients. The resulting formulas are interesting in themselves as constituting an application of the alternating components of connection; in addition, they will be found useful in later developments -- as a matter of fact we have already employed one of them in the proof of Theorem 5.3.

As a preliminary, we list two needed recursion formulas:

$$
L_{d}^{a a}=L_{d}^{a-1 \cdot a^{\prime}}-L_{b}^{a-1 \cdot a} L_{d}^{b}, c_{i}>0 ; L_{d}^{0 a}=\delta_{d}^{a}
$$

(6.2) $L_{a a}^{c}=\frac{A+1}{M-A}\left(L_{A+1 \cdot a}^{c}+L_{A+1 \cdot a}^{b} L_{b}^{c}\right), A=u, u<M ; L_{M a}^{c}=\delta_{a}^{c}$.

These formulas were first found by A. Kawaguchi and later independently by one of the present writers-- see [9, p. 105-108; 4, p.21-29, 5, p.338-339]. They are essentially rules for going from a given rank to a rank one step further removed from tensor rank. In the case of a Greek superscript $c_{\text {. }}$, the tensor rank is that for which $a$ has the minimum value zero, while in the case of a subscript the tensor rank is given by the maximum value $M$. It will be helpful to bear in mind that the operations involved are formally equivalent to intrinsic differentiation except that (6.2) involves the quotient of two binomial coefficients ${ }_{M} C_{A} /{ }_{M} C_{A+1}$. Also, it should be noted that for a given $c, L_{\alpha a}^{c}$ depends on $M$, while $L_{d}^{\alpha a}$ does not.

The components of the tensors $T$ appearing in the following theorems are to be regarded as functions of $t$, the curve parameter of a parameterized arc $C$. The existence of the necessary derivatives is of course to be assumed; and in cases wherein the existence of a solution of the differential equations $I T=0$ is required, the $L$ 's must be regarded as analytic functions of $t$. It should be borne in mind that neither the intrinsic derivative nor the total derivative of $T$ with respect to $t$ involves values of $T$ off of $C$ in their computation. Finally, as before, we shall assign terms containing out-of-range indices the value zero.

Theorem 6.1. If 


$$
I T^{a}=0
$$

and

$$
T^{\alpha a} \text { denotes the extensor } T^{a(\alpha)} \text {, }
$$

then

$$
T^{a a}=T^{b} L_{b}^{a a}
$$

Proof. The case $a=1$ is obvious since

$$
L_{b}^{1 a}=-L_{b}^{a}
$$

accordingly, we proceed by induction. Differentiating

$$
T^{a a}=T^{c} L_{c}^{a a}
$$

replacing $T^{c}$ with $-T^{b} L_{b}^{c}$, and subsequently applying equation (6.1), we obtain successively

$$
T^{a a^{\prime}}=T^{a+1 \cdot a}=T^{c^{\prime}} L_{c}^{a a}+T^{c} L_{c}^{a a^{\prime}}=T^{b}\left(L_{b}^{a a^{\prime}}-L_{c}^{a a} L_{b}^{c}\right)=T^{b} L_{b}^{a+1 \cdot a} .
$$

THEOREM 6.2. If

$$
I T_{a}=0
$$

and

$$
T_{a a} \text { denotes }\left(\begin{array}{c}
M \\
A
\end{array}\right) T_{a}^{\bullet(M-A)},
$$$$
A=c_{\text {, }}
$$

then

$$
T_{a a}=T_{b} L_{a a}^{b}
$$

Method of proof. This theorem may be established by means of the procedure employed in the previous demonstration.

THEOREM 6.3. If

$$
I T^{a b}=0
$$

and

$$
T^{\alpha a \beta b} \text { represents the extensor }\left[\begin{array}{c}
A B \\
M
\end{array}\right] T^{a b(A+B-M)} \text {, }
$$




$$
\begin{aligned}
T^{a b(\gamma)} & =T^{c d} \sum_{\delta=0}^{M}\left(\begin{array}{c}
\gamma \\
\delta
\end{array}\right) L_{c}^{\delta a} L_{d}^{\gamma-\delta \cdot b}, \\
T^{\alpha a \beta b} & =T^{c d} \sum_{\delta=0}^{M}\left\{\begin{array}{l}
A, B \\
M, \delta
\end{array}\right\} L_{c}^{\delta a} L_{d}^{A+B-M-\delta \cdot b} .
\end{aligned}
$$

Proof. Given any value of $t$, say $t_{0}$, the components of $T$ at $t_{0}$, namely $T^{a b}\left(t_{0}\right)$, may be expressed as a sum of prodıcts of pairs of contravariant vectors at $t_{0}$; thus

$$
T^{a b}\left(t_{0}\right)=A^{a} B^{b}+C^{a} D^{b}+\cdots
$$

Here the symbols $A^{a}, B^{b}$, etc., denote fixed real numbers in the coordinate system at hand; and assuming that the $L$ 's and the arc $C$ are analytic, we may define vector fields $A^{a}(t), B^{b}(t)$, etc., along $C$, such that

$$
I A^{a}(t)=0, A^{a}\left(t_{0}\right)=A^{a}, \text { etc. }
$$

The sum

$$
A^{a}(t) B^{b}(t)+C^{a}(t) D^{b}(t)+\cdots
$$

will then have the property that its intrinsic derivative vanishes, and that it takes on the value $T^{a b}\left(t_{0}\right)$ at $t=t_{0}$. But the original equipollent tensor $T^{a b}$ has these properties, and conversely it is uniquely determined by them. Consequently,

$$
T^{a b} \underset{t}{\equiv} A^{a}(t) B^{b}(t)+C^{a}(t) D^{b}(t)+\cdots
$$

To simplify the writing, we shall denote the entire sum in the preceding equation by $A^{a} B^{b}$.

Differentiating $T^{a b} \gamma$ times with respect to $t$, and subsequently employing Theorem 6.1, we get

$$
\begin{aligned}
T^{a b(\gamma)}=\left(A^{a} B^{b}\right)^{(\gamma)} & =\sum_{\delta=0}^{\gamma}\left(\begin{array}{c}
\gamma \\
\delta
\end{array}\right) A^{a(\delta)} B^{b(\gamma-\delta)} \\
& =\sum_{\delta}\left(\begin{array}{l}
\gamma \\
\delta
\end{array}\right) L_{c}^{\delta a} L_{d}^{\gamma-\delta \cdot b} T^{c d} .
\end{aligned}
$$

To obtain the second equation of the theorem, we substitute $A+B-M$ for $\gamma$ and multiply by the coefficient [] inf. $M$, sup. $A, B$; thus 


$$
T^{\alpha a \cdot B b}=\left[\begin{array}{c}
A, B \\
M
\end{array}\right] T^{a b(A+B-M)}=T^{c d} \sum_{\delta}\left\{\begin{array}{l}
A, B \\
M, \delta
\end{array}\right\} L_{c}^{\delta a} L_{d}^{A+B-M-\delta \cdot b} .
$$

Evidently, the range of summation on $\delta$ may be taken to be 0 to $M$ since the coefficient vanishes whenever $\delta>A+B-M$. Whenever $A+B<M, T^{a a \cdot \beta b}$ is zero; in this case the nultiplier of $T^{c d}$ has the value zero by virtue of our convention regarding symbols bearing out-of-range indices.

REMARK. Since $I g^{a b}=0$, we may take $T^{a b}$ to be $g^{a b}$; this special case is the corollary cited in the proof of $\mathrm{ineorem} \tilde{j} .3$.

THEOREM 6.4. If

$$
I T_{b}^{a}=0
$$

and

$$
T_{\beta b}^{\alpha a} \text { denotes }\left(\begin{array}{c}
A \\
B
\end{array}\right) T_{b}^{a(A-B)},
$$

then

$$
\begin{gathered}
\left(\begin{array}{c}
M \\
\Gamma
\end{array}\right) T_{b}^{a(\Gamma)}=T_{d}^{c} R L^{\delta-(M-\Gamma) a} L_{\delta b}^{d}, \\
T_{\beta b}^{\alpha a}=\left(\begin{array}{c}
A \\
B
\end{array}\right)\left(\begin{array}{c}
M-B \\
A-1
\end{array}\right)^{-1} R L^{\delta-(M-A+B)} L \quad \text { (unchanged indices omitted). }
\end{gathered}
$$

Proof. We write $T_{b}^{a}$ in the form $A^{a} B_{b}$, with the understanding that the latter expression represents a sum of products of equipollent vectors. Differentiating this symbolic product $y$ times with respect to $t$, and writing $B_{b}{ }^{(\delta)}$ in the form $\left(\begin{array}{c}M \\ \delta\end{array}\right)^{-1} B_{M-\delta \cdot b}$, provides the relationship

$$
\begin{aligned}
T_{b}^{a\left(\Gamma^{\prime}\right)}=\left(A^{a} B_{b}\right)^{(\Gamma)} & =\sum_{\delta=0}^{\Gamma}\left(\begin{array}{c}
\Gamma \\
\delta
\end{array}\right) A^{a(\Gamma-\delta)}\left(\begin{array}{c}
M \\
\delta
\end{array}\right)^{-1} B_{M-\delta} b \\
& =T_{d}^{c} \sum_{\delta}\left(\begin{array}{c}
\Gamma \\
\delta
\end{array}\right)\left(\begin{array}{c}
M \\
\delta
\end{array}\right)^{-1} L_{c}^{\Gamma-\delta} \cdot a L_{M-\delta}^{d} \cdot b
\end{aligned}
$$

Replacing the binomial coefficients with the equivalent product $\left(\begin{array}{c}M \\ \Gamma\end{array}\right)^{-1}\left(\begin{array}{c}M-\delta \\ M-\end{array}\right)$, and subsequently the dummy index $\delta$ with $M-\delta$, we obtain

$$
T_{b}^{a(\Gamma)}=T_{d}^{c}\left(\begin{array}{c}
M \\
\Gamma
\end{array}\right)^{-1} R L_{c}^{\delta-(M-\Gamma) a} L_{\delta b}^{d}
$$

the first of the relations to be established. We next set $\Gamma=a-\beta$ and get 


$$
T_{\beta b}^{\alpha a}=T_{d}^{c}\left(\begin{array}{c}
A \\
B
\end{array}\right)\left(\begin{array}{c}
M \\
A-B
\end{array}\right)^{-1} K L L
$$

REMARK. Since

$$
\left(\begin{array}{l}
A \\
B
\end{array}\right)\left(\begin{array}{c}
M \\
A-B
\end{array}\right)^{-1}\left(\begin{array}{c}
\delta \\
M-A+B
\end{array}\right)=\left[\begin{array}{c}
A, \Delta \\
M
\end{array}\right]\left(\begin{array}{c}
A+\Delta-M \\
\beta
\end{array}\right)=\left\{\begin{array}{c}
a, \delta \\
M, \beta
\end{array}\right\}
$$

the preceding equation may be written in the form

$$
T_{\beta b}^{a a}=T_{d}^{c} \sum_{\delta=M-A+B}^{M}\left\{\begin{array}{l}
A, \delta \\
M, B
\end{array}\right\} L_{c}^{A+\delta-M-B \cdot a} L_{\delta b}^{d}
$$

We note in passing that there is a pleasing regularity with regard to the pattern of the Creek indices in the right member of (6.3), which may be described as follows: (1) the positive indices on $L_{c}$ appear on top in \{\} , while the negatives are on the bottom; (2) the summation is on the repeated index $\delta$ in $L L$, and starts at the zero of the total Greek superscript and ends at $M$. Whenever such regularity holds, we shall write the \{\} without indices and omit the summation sign. Applying this convention, we rewrite (6.3) in the abridged form

$$
T_{\beta b}^{a a}=T_{d}^{c}\{\} L_{c}^{a+\delta-M-\beta \cdot a} L_{\delta b}^{d}
$$

THEOREM 6.5. If I $T_{a b}=0$, and

$$
T_{a a \cdot \beta b} \text { denotes }\left(\begin{array}{c}
M \\
A B
\end{array}\right) T_{a b}^{\cdots(M-A-B)}
$$

then

$$
\begin{aligned}
T_{\alpha a \cdot \beta b}=T_{c d} \sum_{\delta} \underset{M, A, B}{\left\{\begin{array}{c}
M-\delta, A+\delta \\
A
\end{array} L_{M-\delta \cdot a}^{c} L_{A+B+\delta \cdot b}^{d},\right.} \\
\quad A=c_{i}, B=\beta, \delta: 0 \text { to } M-a-\beta .
\end{aligned}
$$

Proof. As before, we express the tensor as a symbolic product, thus

$$
T_{a b}=A_{a} B_{b}
$$

We then differentiate $\gamma$ times with respect to $t$, and replace the resulting derivatives of $A$ and $B$ by their values expressed as contractions with $L$ (Theorem 6.2). The result is the equality

$$
T_{a \dot{b}}^{\ddot{\bullet}(\gamma)}=T_{c d} \sum_{\delta=0}^{\Gamma}\left(\begin{array}{c}
\Gamma \\
\delta
\end{array}\right)\left(\begin{array}{c}
M \\
\delta
\end{array}\right)^{-1}\left(\begin{array}{c}
M \\
\Gamma-\delta
\end{array}\right)^{-1} L_{M-\delta \cdot a}^{c} L_{M-\Gamma+\delta \cdot b}^{d} \cdot
$$


Finally, we set $\gamma=M-C-\beta$, multiply by $(\underset{A, B}{M})$, employ the numerical equality

$$
\left(\begin{array}{c}
M \\
A, B
\end{array}\right)\left(\begin{array}{c}
\Gamma \\
\Delta
\end{array}\right)(\underset{\Delta}{M})^{-1}(\underset{\Gamma-\Delta}{M})^{-1}=\{\underset{M, A}{M-B}, A+B+\Delta\}, \Gamma=M-A-B,
$$

and the theorem follows.

REMARK. Because of the arbitrariness of the numerical values of $T$ at any one point, we may conclude that the multipliers of $T$ in the expressions for the extensors $T^{a a} \cdot \beta b, T_{\beta b}^{a a}, T_{\alpha a} \cdot \beta b[$ [Theorems 6.3-6.5] are themselves extensors.

REMARK. In case $I T$ vanishes, the $L$ 's and certain semi-contractions (contractions on but one letter of a doublet index) serve to express $T(t+h)$ in terms of $T(t)$. To illustrate, if $I T^{a}=0$, then from Taylor's theorem and Theorem 6.1, we have at once

$$
T^{a}(t+h)=T^{b}(t) \sum_{a} L_{b}^{a a} \frac{(h)^{\alpha}}{c_{n} !}, \quad \quad \alpha: 0 \text { to } \propto .
$$

In the Cartesian case,

$$
L_{c}^{a a}=\delta_{0}^{a} \delta_{a}^{c}
$$

and, of course,

$$
T^{a}(t+h)=T^{a}(t)
$$

7. Contracted products. As a step in rounding out the theory of the metric extensors, and as an implement for certain operations involving these extensors, it is desirable to have a table of their contractions. Several of these contractions yield Kronecker deltas, and thus provide the possibility in certain instances of circumventing the restricting circumstance of the lack of a division process corresponding to multiplication with contraction.

The methods employed in constructing our tables of contractions are not always direct. Consequently, it is necessary that we digress and consider at the outset one or two preliminary matters. The first of these is a formula relating the $L_{\alpha a}^{b}$ associated with a given value of $M$ with the corresponding $L$ associated with $M-\theta$. This formula is a result of repeated application of the relation,

$$
L_{a a}^{* b}=(M / A) L_{A-1 \cdot a}^{b}, \quad A=u,
$$

( See [ 5, p. 338, equation 13.8].), in which the $M$ of $L^{*}$ exceeds that of $L$ by one. Thus if we denote by $U$ the process of lowering by unity the values of $M$ and $a$ in 
$L_{\alpha a}^{b}$, then we may write, successively,

$$
L=\frac{M}{u} U L=\frac{M(M-1)}{u(c-1)} U^{2} L=\left(\begin{array}{c}
M \\
2
\end{array}\right)\left(\begin{array}{c}
\alpha \\
2
\end{array}\right)^{-1} U^{2} L \text {. }
$$

Continuing, we see that the operator $U^{\theta}$ is equivalent to $\left(\begin{array}{c}M \\ \theta\end{array}\right)^{-1}\left(\begin{array}{c}a \\ A\end{array}\right)$; i. e.,

$$
U^{\theta} L_{\alpha a}^{b}=\left(\begin{array}{c}
M \\
\theta
\end{array}\right)^{-1}\left(\begin{array}{c}
A \\
\theta
\end{array}\right) L_{A a}^{b}, \quad A=u .
$$

Here it should be recalled that while $L_{a a}^{b}$ is dependent on the value of $M$ chosen, $L_{b}^{a \alpha}$ is not.

As an application (which will be needed presently) of formula (7.1), we note that the expressions as full range contractions for repeated intrinsic derivatives may be transformed to reduced range contractions for a higher value of $M$. Thus if we denote the order of the intrinsic derivative by $M-\theta$, the $L$ 's involved in the case of contravariant tensors are those associated with $M-\theta$, and may be replaced by the $L$ 's correlated to $M$ if the summation index is written in the form $a-\theta$, instead of $a$, and formula (7.1) is applied. In order to express the association of a quantity with $M-\theta$ ( rather than with $M$ ), we shall write it with a superior-, thus $L^{-}$.. Now, with the notations and procedures indicated, the derivative $I^{M-\theta} A^{b}$ may be transformed as follows:

$$
\begin{aligned}
I^{M-\theta} A^{b} & =\sum_{a=0}^{M-\theta} A^{a(\alpha)} L_{\alpha a}^{b-}=\sum_{a=\theta}^{M} A^{a(\alpha-\theta)} L_{a-\theta \cdot a}^{b-} \\
& =\sum A^{a(\alpha-\theta)} U^{\theta} L_{\alpha a}^{b}=\left(\begin{array}{c}
M \\
\theta
\end{array}\right)^{-1} R A^{a(\alpha-\theta)} L_{\alpha a}^{b} ;
\end{aligned}
$$

that is,

$$
\left(\begin{array}{c}
M \\
\theta
\end{array}\right) l^{M-\theta} A^{b}=R A^{a(\alpha-\theta)} L_{\alpha a}^{b} .
$$

On the other hand, $L_{b}^{\alpha a}$ is independent of $M$, while

$$
B_{a a}\left[=\left(\begin{array}{c}
M \\
A
\end{array}\right) B_{a}^{\cdot(M-A)}\right]
$$

follow's the companion formula to (7.1), namely,

$$
U^{\theta} B_{a a}=\left(\begin{array}{c}
M \\
\theta
\end{array}\right)^{-1}\left(\begin{array}{c}
A \\
\theta
\end{array}\right) B_{A a} \cdot
$$

Consequently, the contraction expressing a higher order intrinsic derivative of a covariant vector $B_{b}$ may be altered, thus: 


$$
I^{M-\theta} B_{b}=\sum_{a=0}^{M-\theta} B_{a a}^{-} L_{b}^{a a}=\sum_{a=\theta}^{M} B_{a-\theta \cdot a}^{-} L_{b}^{\alpha-\theta \cdot a}=\left(\begin{array}{c}
M \\
\theta
\end{array}\right)^{-1} R B_{a a} L_{b}^{\alpha-\theta} \cdot a
$$

or,

$$
\left(\begin{array}{c}
M \\
\theta
\end{array}\right) I^{M-\theta} B_{b}=R B_{\alpha a} L_{b}^{a-\theta \cdot a}
$$

Similarly, the full range contraction which yields a higher order intrinsic derivative of the mixed tensor $T_{b}^{a}$ may be converted into a form involving one or nore reduced contractions. These expressions involving reduced contractions can be used to evaluate the contractions of two L's. Accordingly, we shall develop one in detail. Starting with the intrinsic derivative of order $M-\theta$ expressed as a full range contraction, namely,

$$
I^{M-\theta} T_{b}^{a}=\sum T_{\delta d}^{\gamma c} L_{b}^{\delta d} L_{\gamma c}^{a-}, \quad \quad \text { sum } \gamma, \delta: 0 \text { to } M-\theta
$$

we replace the dummy index $y$ with $y-\theta$, making the new range for $\gamma$ from $\theta$ to $M$. The symbol $L_{\gamma c}^{a-}$ must be replaced with $L_{\gamma-\theta \cdot c}^{a-}$, which in turn may be written

$$
U^{\theta} L_{\gamma c}^{a} \text { or }\left(\begin{array}{c}
M \\
\theta
\end{array}\right)^{-1}\left(\begin{array}{c}
\Gamma \\
\theta
\end{array}\right) L_{\Gamma c}^{a}
$$

Thus we have the relationship

$$
\left(\begin{array}{c}
M \\
\theta
\end{array}\right) I^{M-\theta} T_{b}^{a}=\sum_{\delta=0}^{M-\theta} R T_{\delta d}^{\gamma-\theta \cdot c} L_{b}^{\delta d} L_{\gamma c}^{a}
$$

With formulas (7.2), (7.4), (7.5) and Theorems 6.1, 6.2,6.3 as a foundation, we are now ready to consider certain of the contracted products of our metric extensors. Our first proposition of this series may be stated as follows:

THEOREM 7.1. $R L_{d}^{a-\theta} \cdot a L_{a a}^{c}=\delta_{\theta}^{M} \delta_{d}^{c}$.

Proof. If $A^{a}$ is any equipollent vector, then, by Theorem 6.1 ,

$$
A^{a(a-\theta)}=L_{b}^{a-\theta \cdot a} A^{b} .
$$

Multiplication by $\left(\begin{array}{l}a \\ \theta\end{array}\right)$ and $L_{a a}^{c}$ with subsequent contraction yields

$$
A^{b} R L_{b}^{a-\theta \cdot a} L_{a a}^{c}=R A^{a(a-\theta)} L_{a a}^{c}=\left(\begin{array}{c}
M \\
\theta
\end{array}\right) I^{M-\theta} A^{c}=\delta_{\theta}^{M} A^{c} .
$$

Since the values of $A^{b}$ at $t=t_{0}$ may be assigned arbitrarily, we conclude that if $\theta \neq M$, then 


$$
R L L=0 \text {. }
$$

When $\theta=M$, we have

$$
R L L=\delta_{b}^{a} \delta_{a}^{c} \text {, }
$$

and the theorem is established.

COROLLARY. $L_{d}^{\alpha a} I_{a a}^{c}=\delta_{0}^{M} \delta_{d}^{c}$.

A formula similar to that of Theorem 7.1 holds for cross summations, as witness:

THEOREM 7.2. $R L_{c}^{a-\theta \cdot a} L_{a b}^{c}=\delta_{\theta}^{M} \delta_{b}^{a}$.

Proof. This theorem follows at once from Theorem 6.4 by taking $T_{b}^{a}=\delta_{b}^{a}$ and replacing $\Gamma$ with $M-\theta$.

Corollary. $L_{c}^{a a} L_{a b}^{c}=\delta_{0}^{M} \delta_{b}^{a}$.

Evidently, we can derive companion theorems to Theorem 7.2 by taking $T^{a b}$ and $T_{a b}$ in Theorems 6.3 and 6.5 to be $g^{a b}$ and $g_{a b}$, respectively. Thus we have:

Theовем 7.3. $g^{\alpha a \cdot \beta b}=g^{c d} \sum\left\{\begin{array}{l}A B \\ M\end{array}\right\} L_{c}^{\delta a} L_{d}^{A+B-M-\delta \cdot b}$, sum $\delta: 0, M$;

$$
\begin{aligned}
g_{\alpha a} \cdot \beta b & =g_{c d} \sum\left\{\begin{array}{c}
M-\delta, A+B+\delta \\
M, A, B
\end{array}\right\} L_{M-\delta \cdot a}^{c} L_{A+B+\delta \cdot b}^{d}, \\
& A=\alpha, B=\beta \text {, sum } \delta: 0, M-a-\beta .
\end{aligned}
$$

REMARK. These theorems relate the interchange extensors (script g's) to the alternating components of connection.

The contractions of the interchange extensors with the direct and alternating components of connection, as we have seen, convert excontravariance to excovariance, and vice versa. Accordingly, we turn our attention to the direct components of connection, and consider the contractions $g L$ and $g g$. In order to evaluate the former, we first observe that since the $\vec{\rho}^{a}$ constitute a set of quantities (directed strokes) that transform contravariantly, their intrinsic derivatives $I^{\alpha} \vec{\rho}^{a}$ also possess this property. Furthermore, these derivatives may be computed by equations of the type (7.2), (7.4). Thus we may write

$$
\left(\begin{array}{c}
M \\
\theta
\end{array}\right) I^{M-\theta} \vec{\rho}^{b}=R \vec{\rho}^{a(a-\theta)} L_{a a}^{b}
$$


and

$\left(\begin{array}{c}M \\ \theta\end{array}\right) I^{M-\theta} \vec{\rho}_{b}=R \vec{\rho}_{\alpha a} L_{b}^{\alpha-\theta \cdot a}, \quad$ where $\vec{\rho}_{\alpha a}$ denotes $\vec{\rho}_{; \alpha a}^{(M)}$ or $\left(\begin{array}{c}M \\ A\end{array}\right) \vec{\rho}_{a}^{\cdot(M-A)}$.

Multiplication of these by $\vec{\rho}_{c}$, and $\vec{\rho}^{c}$ - respectively gives the following:

THEOREM 7.4. $R g_{c}^{a-\theta \cdot a} L_{a a}^{b}=\left(\begin{array}{c}M \\ \theta\end{array}\right) \vec{\rho}_{c} \cdot l^{M-\theta} \vec{\rho}^{b}$,

$$
R L_{b}^{a-\theta \cdot a} g_{a a}^{c}=\left(\begin{array}{c}
M \\
\theta
\end{array}\right) \vec{\rho}^{c} \cdot I^{M-\theta} \vec{\rho}_{b} \cdot
$$

REMARK. The right members of the preceding equalities determine the scalar components of the parts of the higher-order intrinsic derivatives of the base vectors which belong to the tangent space $T_{N}$. In case $\theta=M-1$, the contractions reduce essentially to contractions of the $L$ 's, and we conclude that $I \vec{\rho}^{a}$ and $I \vec{\rho}_{a}$ either vanish or are perpendicular to $R_{N}$.

This last property can be established directly by applying the operator $\vec{\rho}_{d}$. to the following chain:

$$
I \vec{\rho}^{a}=\vec{\rho}^{a^{\prime}}+\vec{\rho}^{b}\left\{\begin{array}{c}
a \\
b c
\end{array}\right\} x^{c^{\prime}}=\vec{\rho}^{a^{\prime}}+\vec{\rho}^{b} \vec{\rho}^{a} \cdot \vec{\rho}_{b}^{\prime}=\vec{\rho}^{a^{\prime}}-\vec{\rho}^{b}\left(\vec{\rho}^{a} \cdot \vec{\rho}_{b}\right) .
$$

Thus the geometric meaning of $l \vec{\rho}^{a}$ is that it is the part of $\vec{\rho}^{a^{\prime}}$ that does not belong to the tangent space $T_{N}$. Evidently, the set of directed strokes $I \vec{\rho}^{a}$ transforms contravariantly, while the $l \vec{\rho}^{a}$ are covariant. In the case of a completely flat space we have

$$
I \vec{\rho}_{a}=0
$$

since in such space we have

$$
\Delta \vec{\rho}=\left(\vec{\rho}_{b} \vec{\rho}^{b}\right)_{\text {at } 0} \cdot \Delta \vec{\rho}
$$

for $\Delta \vec{\rho}=\vec{\rho}(P)-\vec{\rho}(0)$ with, if we like, 0 fixed and $P$ variable. Differentiating this last equation with respect to $x^{a}$ and $t$ we get, successively,

$$
\vec{\rho}_{a}=\left(\vec{\rho}_{b} \vec{\rho}^{b}\right)_{\text {at } 0} \cdot \vec{\rho}_{a} \text { and } \vec{\rho}_{a}^{\prime}=\left(\vec{\rho}_{b} \vec{\rho}^{b}\right)_{\text {at } 0} \cdot \vec{\rho}_{a}^{\prime} \text {. }
$$

If we now evaluate $\vec{\rho}_{a}^{\prime}$ at 0 , we obtain, on transposing, the conclusion $I_{\rho}=0$. In general, $I \vec{\rho}_{a}$ is the part of $\vec{\rho}_{a}^{\prime}$ that does not lie in $T_{N}$. Furthermore we observe, in passing, that in the case of a completely flat space all derivatives of the base vectors lie in $T_{N}$.

The interpretation of the contractions $g_{b}^{\alpha a} g_{\alpha a}^{c}$ and $g_{b}^{a a} g_{a d}^{c}$ may be obtained readily by transforming the latter as follows:

$$
g_{b}^{a a} g_{a d}^{c}=\vec{\rho}_{b} \cdot \vec{\rho}^{a(a)} \vec{\rho}_{a d} \cdot \vec{\rho}^{c}=\vec{\rho}_{b} \cdot\left(\vec{\rho}^{a} \vec{\rho}_{d}\right)^{(M)} \cdot \vec{\rho}^{c},
$$


with

$$
\vec{\rho}_{a d}=\left(\begin{array}{c}
M \\
A
\end{array}\right) \vec{\rho}_{d}^{\cdot}(M-A)
$$

Thus $g_{b}^{a} a g_{a d}^{c}$ is the scalar component of the part of the $M$ th derived dyad $\left(\vec{\rho}^{a} \vec{\rho}_{b}\right)^{(M)}$ that belongs to the space.

8. The tensor $D_{b c d^{*}}^{a}$. The intrinsic derivatives based on the direct and alternating extensors $g$ and $L$ coincide for 11 zero or unity. Furthermore, they coincille for all values of $M$ if the space is completely flat since in this latter case all derivatives of the base vectors and therefore all derivatives of $V^{a} \vec{\rho}_{a}$ lie in the space. Accordingly, the difference between the second intrinsic derivatives of $V^{a}$ should lead to a distinction between completely flat spaces and other varieties.

Instead of limiting our attention to the one-parameter case, it will perhaps be more interesting to carry out the investigation for parameterized surfaces in $R_{N}$. Consequently, we now set each of the variables $x^{a}$ of our immersed space $R_{N}$ equal to a function $x^{a}(u, v)$ of two parameters. Thus the radius vector of our enveloping Euclidean space is now a function of $u$ and $v$ through the $x$ 's. Symbolically,

$$
\vec{\rho}=\vec{\rho}[x(u, v)]
$$

The direct metric extensors $g_{\beta b}^{a}$ are defined formally as before:

$$
g_{\beta b}^{a}=\vec{\rho}^{a} \cdot \vec{\rho}_{\beta b}, \vec{\rho}_{\beta b}=\vec{\rho}_{; \beta b}^{(M)}=\left(\begin{array}{c}
M \\
B
\end{array}\right) \vec{\rho}_{b}^{(M-B)} .
$$

The difference is that $M$ and $\beta$ now represent the matrices $\left(M_{1}, M_{2}\right),\left(\beta_{1}, \beta_{2}\right)$, and the binomial symbol is the matrix binomial coefficient defined in $\$ 2$.

The mixed second-order direct intrinsic derivative is given by the contraction $V^{b(\beta)} g_{\beta b}^{a}$, with $M=\left(\begin{array}{ll}1 & 1\end{array}\right)$, so that the range of $\beta$ is $\left(\begin{array}{ll}0 & 0\end{array}\right),\left(\begin{array}{ll}0 & 1\end{array}\right),\left(\begin{array}{ll}1 & 0\end{array}\right),\left(\begin{array}{ll}1 & 1\end{array}\right)$. Evidently, since the Leibnitz rule for the differentiation of products holds for "matrix primes", we may write, for all admissible matrices $M$,

$$
V^{b(\beta)} g_{\beta b}^{a}=\left(V^{b \beta} \vec{\rho}_{\beta b}\right) \cdot \vec{\rho}^{a}=\left(V^{b} \vec{\rho}_{b}\right)^{(M)} \cdot \vec{\rho}^{a},
$$

and conclude as before that the direct derivatives give the part of $\vec{\sigma}^{(M)},(\vec{\sigma}=$ $V^{a} \vec{\rho}_{a}$ ) that belong to the tangent space $T_{N}$.

In order to compute the alternating intrinsic derivative $I^{01} I^{10} V^{a}$, we first write

$$
\left.I^{10} V^{d}=V^{b(\beta)} g_{\beta b}^{d}, M=\left(\begin{array}{ll}
1 & 0
\end{array}\right),=V^{d\left(\begin{array}{ll}
1 & 0
\end{array}\right)}+V^{b} \vec{\rho}^{d} \cdot \vec{\rho}_{b}^{(10}\right)=U^{d}
$$


and then

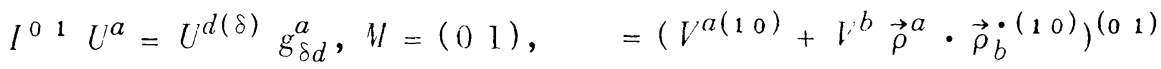

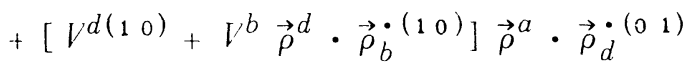

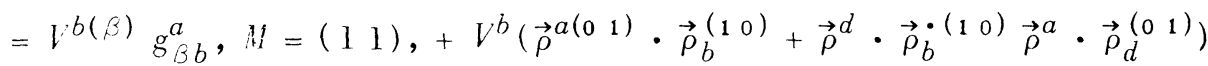

$$
\begin{aligned}
& =V^{b(\beta)} g_{\beta b}^{a}, M=\left(\begin{array}{ll}
1 & 1
\end{array}\right),+V^{b} x^{c(10)} x^{d(01)}\left(\vec{\rho}_{b c} \cdot \vec{\rho}_{d}^{a}+\left\{\begin{array}{c}
e \\
b c
\end{array}\right\}\left\{\begin{array}{c}
a \\
e d
\end{array}\right\}\right) \text {. }
\end{aligned}
$$

Consequently, if we denote the parentiesis by $D_{b c d}^{\bullet a}$, we have

$$
I^{01} I^{10} V^{a}-V^{b(\beta)} g_{\beta b}^{a}=V^{b} x^{c(10)} x^{d\left(\begin{array}{ll}
0 & 1
\end{array}\right)} D_{b c d}^{\cdots a},
$$

and this difference vanishes for all vectors $V$ and all parameterized surfaces only in case the tensor $D_{\vec{b} c d}^{\vec{d}}$ is zero.

This tensor ${ }^{2}$ in one form or another and in particular certain of its associated quantities occur in numerous places in the literature. The : iiemann-Christoffel tensor, for example, is a skew-symmetric part of $2 D$, since $\nu_{b c} \ddot{d}^{a}-\nu_{b \dot{d}} \ddot{b}^{a}=$ Ricd

Summarizing this and our preceding results, we have the general conclusion that the metric extensors do play a fundamental role in the geometry of the immersed $i_{N}$.

2 The present writers are indebted to Vaclav Hlavaty for historical information pertaining to $D$ and its relata.

\section{REFERENCES}

1. H. V. Craig, On a generalized tangent vector, Amer. J. Math. 57 (1935), 457-462.

2. - On tensors relative to the extended coordinate transformation, Amer. J. Math. 59 (1937), 764-774.

3. - Vector and ten sor analysis, New York, 1943.

4. - On the structure of certain tensors, Math. Mag. 1 (1947), 21-30.

5. - On the structure of intrinsic derivatives, Bull. Amer. Math. Soc. 53 (1947), 332-342.

6. H. V. Craig and W. T. Guy, Jr., Jacobian extensors, Amer. J. Math. 72 (1950), 229246.

7. Philip Franklin, Derivatives of higher order as single limits, Bull. Arer. Math. Soc. 41 (1935), 573.

8. A. Kawaguchi, Eine Verallgemeinerung von Extensoren, Monatsheften fur Mathematic und Physik, 48 (1939), 329-339.

9. - Die Differentialgeometrie höherer Ordnung I. Erweiterte Koordinatentransformationen und Extensoren, Journal of the Faculty of Science, Hokkaido Imperial University (I), 9 (1940). 


\section{PACIFIC JOURNAL OF MATHEMATICS}

\section{EDITORS}

\author{
R. M. Foeinson \\ University of California \\ Berkeley 4, California \\ E. HEWITt \\ University of Washington \\ Seattle 5, Washington
}

R. P. DILWOR TH

California Institute of Technology

Pasadena 4, California

E. F. BECKENBACH

University of California

Los Angeles 24, California

\section{ASSOCIATE EDITORS}

$\begin{array}{llll}\text { H. BUSEMANN } & \text { P. R. HALMOS } & \text { BøRGE JESSEN } & \text { J. J. STOKER } \\ \text { HERBERT FFDERER } & \text { HEINZ HOPF } & \text { PAUL LÉVY } & \text { E. G. STR AUS } \\ \text { MARSHALL IIALI } & \text { R. D. JAMES } & \text { GEORGE PÓLYA } & \text { KỎSAKU YOSIDA }\end{array}$

\section{SPONSORS}

UNIVERSITY OF BRITISH COLUMBIA CAIIFORNIA INSTITUTE OF TECHNOLOGY UNIVERSITY OF CALIFORNIA, BERKELEY UNIVERSITY OF CAILIFORNIA, DAVIS UNIVERSITY OF CALIFORNIA, LOS ANGELES UNIVERSITY OF CALIFORNIA, SANTA BARBARA UNIVERSITY OF NEVADA OREGON STATE COLLEGE UNIVERSITY OF OREGON
UNIVERSITY OF SOUTHERN CALIFORNIA STANFORD RESEARCH INSTITUTE STANFORD UNIVERSITY WASHINGTON STATE COLLEGE UNIVERSITY OF WASHINGTON

AMERICAN MATHEMATICAL SOCIETY NATIONAL BUREAU OF STANDARDS, INSTITUTE FOR NUMERICAL ANALYSIS

$$
\begin{gathered}
\text { Vari-Type Composition by } \\
\text { Elaine Barth } \\
\text { Delores Wierman } \\
\text { With the cooperation of } \\
\text { E. F. Beckenbach } \\
\text { E. G. Straus }
\end{gathered}
$$

Printed in the United States of America by Edwards Brothers, Inc., Ann Arbor, Michigan 


\section{Pacific Journal of Mathematics}

\section{Vol. 3, No. 1 \\ March, 1953}

Herbert Busemann, Volume in terms of concurrent cross-sections ......... 1

L. Carlitz, Some special equations in a finite field ................. 13

Homer V. Craig and Billie Braden Townsend, On certain metric

extensors ....................................... 25

Philip J. Davis and Henry Pollak, Linear functionals and analytic

continuation problems ............................. 47

Jacob C. E. Dekker, The constructivity of maximal dual ideals in certain

Boolean algebras ................................. 73

Harley M. Flanders, The norm function of an algebraic field extension .... 103

Marshall Hall, Subgroups of free products . . . . . . . . . . . . . . . . . 115

Israel (Yitzchak) Nathan Herstein, Finite multiplicative subgroups in

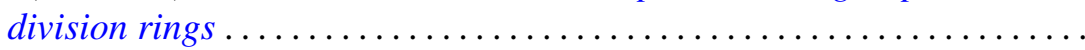

Joseph Lawson Hodges, Jr. and Murray Rosenblatt, Recurrence-time moments in random walks ............................ 127

Alfred Horn, The normal completion of a subset of a complete lattice and lattices of continuous functions ........................ 137

Fulton Koehler, Estimates for the errors in the Rayleigh-Ritz method...... 153

M. H. Martin, The Monge-Ampère partial differential equation

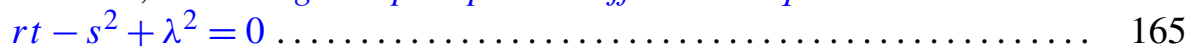

John E. Maxfield, Normal k-tuples ......................... 189

Jack E. McLaughlin, Structured theorems for relatively complemented lattices............................................ 197

William H. Mills, A system of quadratic Diophantine equations ......... 209

T. S. Motzkin, Ernst Gabor Straus and F. A. Valentine, The number of farthest points ................................. 221

G. Power, Forces on the boundary of a dielectric ............... 233

Ralph Gordon Selfridge, Approximations with least maximum error....... 247 